\title{
CARRERAS DE TRAIL RUNNING Y MARCHAS POR MONTAÑA EN ESPAÑA. NÚMERO, EVOLUCIÓN E INCIDENCIA SOBRE RED NATURA 2000
}

\section{Trail running in Spain. Number, evolution and incidence in Natura 2000}

\author{
Estela Inés Farias-Torbidoni1 ${ }^{1,2,}$; Jordi Seguí Urbaneja ${ }^{1,2}$, Rafel Ferrer ${ }^{1}$, Víctor Dorado ${ }^{1}$ \\ ${ }^{1}$ Institut Nacional d'Educació Física de Catalunya (INEFC). Universidad de Lleida. Lleida. España \\ ${ }^{2}$ Miembro del grupo de investigación GISEAFE \\ Identificador ORCID de los autores y e.mail \\ Estela Inés Farías-Torbidoni: https://orcid.org/0000-0002-7541-8140. E-mail: efarias@inefc.es \\ Jordi Seguí Urbaneja: https://orcid.org/0000-0002-5174-4355. E-mail: jsegui@inefc.es \\ Rafel Ferrer: https://orcid.org/0000-0002-0263-220X. E-mail: Ferrer.rafel@gmail.com \\ Victor Dorado: https://orcid.org/0000-0002-7253-8083.E-mail: victordorado90@gmail.com \\ *Autor corresponsal
}

Recibido: 15-09-2017. Aceptado: 9-01-2018. Fecha de publicación on-line: 05/03/2018

Citation / Cómo citar este artículo: Farias-Torbidoni, E.I., Seguí Urbaneja, J., Ferrer, R., Dorado V. (2018). Carreras de trail running y marchas por montaña en España. Número, evolución e incidencia sobre la Red Natura 2000. Pirineos, 173, e034. https://doi.org/10.3989/pirineos.2018.173001

RESUMEN: Siendo evidentes las problemáticas que se pueden derivar del incremento del número de eventos deportivos que se desarrollan en el medio natural y, especialmente, de aquellos que han evidenciado un notable incremento, como es el caso de las Carreras de Trail running y Marchas por montaña (impactos ambientales derivados de su práctica, consolidación de estos espacios como lugar de entrenamiento, masificación, etc.), es objeto de este artículo: 1) identificar el número, evolución y distribución territorial de la organización y desarrollo de Carreras de Trail running y Marchas por montaña en el territorio español, 2) estudiar el grado de incidencia de estos eventos sobre los diferentes Espacios Naturales Protegidos pertenecientes a Red Natura 2000 y 3) analizar algunas de las características principales de estos últimos. Mediante la sistematización y posterior georeferenciación de los 1901 eventos registrados en el año 2015, se llegó a identificar, en términos generales, un predominio de la tipología de carreras a pie, con una tasa de incremento interanual en los últimos cinco años en torno al 8\%, y una distribución, en relación con las diferentes Comunidades Autónomas, muy hetereogénea. En términos específicos, y con un margen de error de $+5 \%$, un grado de incidencia global en torno al $86 \%$. El mes de realización, el número de recorridos y la presión intra Comunidad, fueron algunas de las escasas características que se pudieron analizar en relación al conjunto de las 1624 Carreras de Trail running y Marchas por montaña que transcurren por algunos de los diferentes Espacios Naturales Protegidos incluidos en Red Natura 2000.

PALABRAS CLAVE: Carreras de Trail running, Marchas por montaña, características, evolución, impacto, Red Natura 2000. 


\begin{abstract}
The problems arising from the increase number of sporting events held in the natural environment are evident, especially Trail Running events in Protected Natural Areas (e.g., post event environmental damage, consolidation of these Protected Natural Areas as a training arena, widespread growth). Hence, there is a need to provide insigts to this phenomenon. This work has three main objectives: 1) to identify the number of events, its evolution and territorial distribution of Trail running in Spain, 2) to study the incidence of these events on the different Protected Natural Areas belonging to Natural 2000, and 3) to analyze the main characteristics of the incidence on the Protected Natural Areas. Systematizing and georeferencing the 1901 events registered on the website runedia.com, in 2015, we identified a predominance of foot modality, with an $8 \%$ of yearly increase rate, and a very heterogeneous distribution by regions. With a margin of error of $5 \%$, we were able to establish a global incidence rate of around $86 \%$. The month, the number of tracks and the intra region pressure, were some of the few characteristics that we could analyze in relation to the 1624 Trail Running events which took place in some of the Natura 2000 areas.
\end{abstract}

KEY WORDS: Trail running, caracteristics, evolution, impact, Natura 2000.

\section{Introducción}

Son numerosas las voces y las evidencias que demuestran la consolidación del uso del medio natural como escenario de prácticas físico deportivas, y también la aparición de nuevas tendencias de consumo y, en consecuencia, de negocio, vinculadas al incremento en la organización de eventos deportivos en el medio natural (FernándezArroyo, 2015; Farías, 2015; Farías et al., 2016).

En este sentido, está claro que el medio natural ha pasado de ser un espacio deportivo restringido a grandes especialistas y a personas muy preparadas físicamente, con un gran respeto y sensibilidad hacia el medio, para convertirse en un escenario de práctica menos selectiva y abierta a cualquier tipo de persona que desea iniciarse en la práctica deportiva en cualquiera de sus múltiples modalidades - recreativa, amateur, profesional - (Martos, 2002; Puchan, 2005; Granero \& Baena, 2010). Y nunca mejor dicho, iniciarse. Según datos extraídos de estudios como los llevados a cabo por Bataller et al., 2014 o Farías et al., 2015), entre un 18-22\% de los corredores de carreras (de o por) montaña o Trail running se han iniciado deportivamente mediante esta modalidad.

En este punto, vale la pena destacar, teniendo en cuenta la disertación de la nomenclatura utilizada para definir esta práctica o actividad deportiva (Seguí et al., 2016), a efectos de este artículo, se utilizará el término Trail running como genérico, para referirse indistintamente a carreras de montaña (especialidad deportiva oficialmente reconocida por la Real Federación Española de Atletismo -RFEA-); carreras por montaña (especialidad deportiva oficialmente reconocida por la Federación Española de Deportes de Montaña y Escalada -FEDME-) y carreras de velocidad por montaña (especialidad deportiva oficialmente reconocida por la Federación Catalana de Entidades de Excursionismo -FEEC-). A su vez se considerará, también, las Marchas por montaña como una disciplina o práctica diferente a la del Trail running, aun no siendo esta una modalidad deportiva oficialmente reconocida.

Es en este contexto, donde la presión que ejercen las actividades deportivas que nos ocupan sobre el medio natural en general, y sobre los Espacios Naturales Protegidos (ENP) en particular, ponen en evidencia la necesidad de una gestión activa en torno a la consecución de una adecuada compatibilización de usos, tanto en lo que respecta a la minimización de los impactos ambientales derivados, como en la sensibilización de un correcto uso del medio natural (Newsome et al., 2011; Oñorbe, 2014a). Sin ir más lejos, iniciativas como las llevadas a cabo recientemente a nivel nacional y autonómico por EuroparcEspaña o Fundació Món Rural, centradas, respectivamente, en la elaboración de sendas guías o códigos de buenas prácticas para la organización de carreras de Trail running y Marchas por montaña: Guía de buenas prácticas para el desarrollo de carreras por montaña en espacios naturales protegidos (Europarc-España, 2016) y Codi de bones pràctiques en l'organització i celebració de curses $i$ marxes per muntaya (Fundació Món Rural, 2017), son una buena muestra de ello.

En este sentido, resulta evidente que el incremento y la popularización del desarrollo de eventos deportivos en el medio natural, a pesar de ser iniciativas con un importante potencial de beneficios positivos a diferentes niveles: individual (ej. incremento de la práctica de actividades físico-deportivas, oportunidades para acercarse al medio natural, etc.); social (ej. posibilidad de una mayor sociabilización, etc.); medioambiental (ej. revalorización del medio natural y rural como tal en el caso de una buena gestión del mismo, etc.) o económico (ej. estímulo en el desarrollo regional y económico de determinados lugares, etc.), entre otros, también son susceptibles de generar impactos negativos. Publicaciones como las llevadas a cabo por Ewert et al. (2006), Burgin \& Hardiman (2012), Newsome \& Lacroix (2012), Newsome (2014), a nivel internacional, o por FEDME (2011) y Farías (2015), a nivel nacional, evidencian la aparición de ciertos tipos de problemáticas.

Concretamente, en palabras de Newsome (2014:2), la organización de eventos deportivos que se desarrollan en entornos naturales no tan sólo plantean cuestiones relacionadas con los impactos biofísicos y sociales, sino también con la capacidad de gestión para supervisar adecuadamente esos eventos y la conveniencia o no del desarrollo de tales actividades organizadas dentro del contexto de los ENP.

En general, los impactos ambientales que pueden llegar a provocar la organización de este tipo de eventos no dependen de un solo aspecto, como lo podría ser las ca- 
racterísticas intrínsecas de la actividad practicada (movilidad, requerimientos técnicos, necesidades de equipamientos específicos, interconectividad, etc.), sino que dependen de un continuo de factores como los relacionados con el medio natural en el cual se desarrollan (capacidad de acogida física, social y ecológica, plasticidad o capacidad de resilencia del medio, existencia o no de especies protegidas, presencia de ecosistemas especialmente sensibles que pueden ser afectadas, etc.), las características de los practicantes que las practican (experiencia previa, nivel de preparación física, grado de concienciación y compromiso hacia el respeto de medio natural, etc.) o la forma en que estas son practicadas (número de participantes por evento, distribución temporal y espacial, grado de organización, nivel de implantación de la actividad en la zona o región, etc.), entre otros.

De acuerdo a la consulta de diversas fuentes bibliográficas tales como: Cole (1990), Leung \& Marion (2000), Cater et al. (2008), Pickering (2010), Newsome (2014), Oñorbe (2015), algunos de los principales impactos ambientales directos que se pueden derivar del desarrollo de este tipo de eventos, agrupados según los diferentes componentes, podrían ser:

- Sobre la vegetación: reducción de la altura y vigor de la vegetación, alteración de la composición, pérdida de cobertura vegetal, introducción de especies exóticas.

- Sobre el suelo: pérdida de materia orgánica, desplazamiento del suelo, compactación del suelo, pérdida de la capacidad de infiltración.

- Sobre el agua: incremento de la turbidez del agua, incremento de la entrada de nutrientes, introducción de organismos patógenos, alteración de la composición del agua.

- Sobre la fauna: degradación o pérdida de hábitats, perturbación de la fauna, modificación del comportamiento habitual, desplazamiento a otros hábitats.

- Otros impactos: perturbaciones por ruido, abandono de residuos, deposiciones de los participantes, marcaje y no retirada de señales, etc.

En esta línea de trabajos, según las recomendaciones recogidas en la Guía de buenas prácticas para el desarrollo de carreras por montaña publicado por Europarc-España (2016), tres son los ámbitos de actuación a considerar en el avance de una mayor compatibilización de usos vinculados a los eventos deportivos en los ENP: 1) sobre las entidades organizadoras de la prueba, 2) sobre las administraciones responsables del espacio y 3) sobre los participantes.

De esta manera, y teniendo en cuenta que una gestión más eficaz y eficiente por parte de las administraciones y agentes responsables es necesario, sin lugar a dudas, disponer de datos básicos inherentes a la organización de este tipo de eventos, tales como, el número, la evolución, la incidencia de este tipo de eventos sobre los diferentes ENP entre otras cuestiones. Por ello, y con el objetivo de aportar dichos datos, son objetivos de este artículo:
1) identificar algunas de las principales características de estos eventos, 2) establecer el grado de incidencia de los mismos sobre los diferentes ENP incluidos en Red Natura 2000 y 3) analizar algunas de las características principales de estos últimos.

\section{Metodología}

\section{1. Área de estudio}

El estudio se llevó a cabo teniendo en cuenta, por un lado, la totalidad del territorio español, y por otro, la red de ENP incluidos en Red Natura 2000. En este sentido, cabe destacar que Red Natura 2000 es una red ecológica europea de áreas de conservación de la biodiversidad que incluye la comprensión de dos tipos de zonas: Zonas Especiales de Conservación (ZEC), establecidas de acuerdo con la Directiva Hábitat, y Zonas de Especial Protección para las Aves (ZEPA), designadas en virtud de la Directiva Aves. Por definición, Red Natura 2000 es una red europea de espacios naturales que tiene por objetivo hacer compatible la protección de las especies y los hábitats naturales y seminaturales con la actividad humana que se desarrolla en estos, haciendo que se mantenga un buen estado de conservación de los habitats y especies y así evitar su deterioro.

En la actualidad, un total de 2.111 ENP protegidos conforman la Red Natura 2000 de España: 1.467 Lugares de Importancia Comunitaria (LIC) y 644 Zonas de Especial Protección para las Aves (ZEPA), que con una superficie total de unos de $210.000 \mathrm{~km}^{2}$. De esa extensión total, más de $137.000 \mathrm{~km}^{2}$ se corresponden con superficie terrestre, lo que representa aproximadamente un $27 \%$ del territorio español, y unos $72.500 \mathrm{~km}^{2}$, con superficie marina (Figura 1).

\subsection{Procedimiento}

La metodología de estudio empleada en la realización de este estudio se basó en la sistematización y análisis de datos secundarios correspondientes a la base de datos de las carreras de Trail running y Marchas por montaña en España registradas durante el año 2015. En este sentido cabe destacar, que a pesar del intento de poder contar, para la realización de este estudio, con las bases de datos correspondientes a los calendarios oficiales de Carreras de la RFEA, de la FEDME, de la FEEC y de Runedia (web especializada en la calendarización de eventos deportivos y una de las más populares y extensas del sector del Trail running), solo fue posible basar el estudio en esta última. Mientras que las tres primeras, RFEA, FEDME y FEEC, no respondieron, Runedia (runedia.com) si lo hizo, facilitando el calendario de eventos del año 2015. De aquí que los resultados que se exponen en este artículo fueron elaborados a partir de la sistematización y análisis de la base de datos correspondiente a Runedia para el año 2015. Al respecto, cuatro fueron las fases tenidas en cuen- 


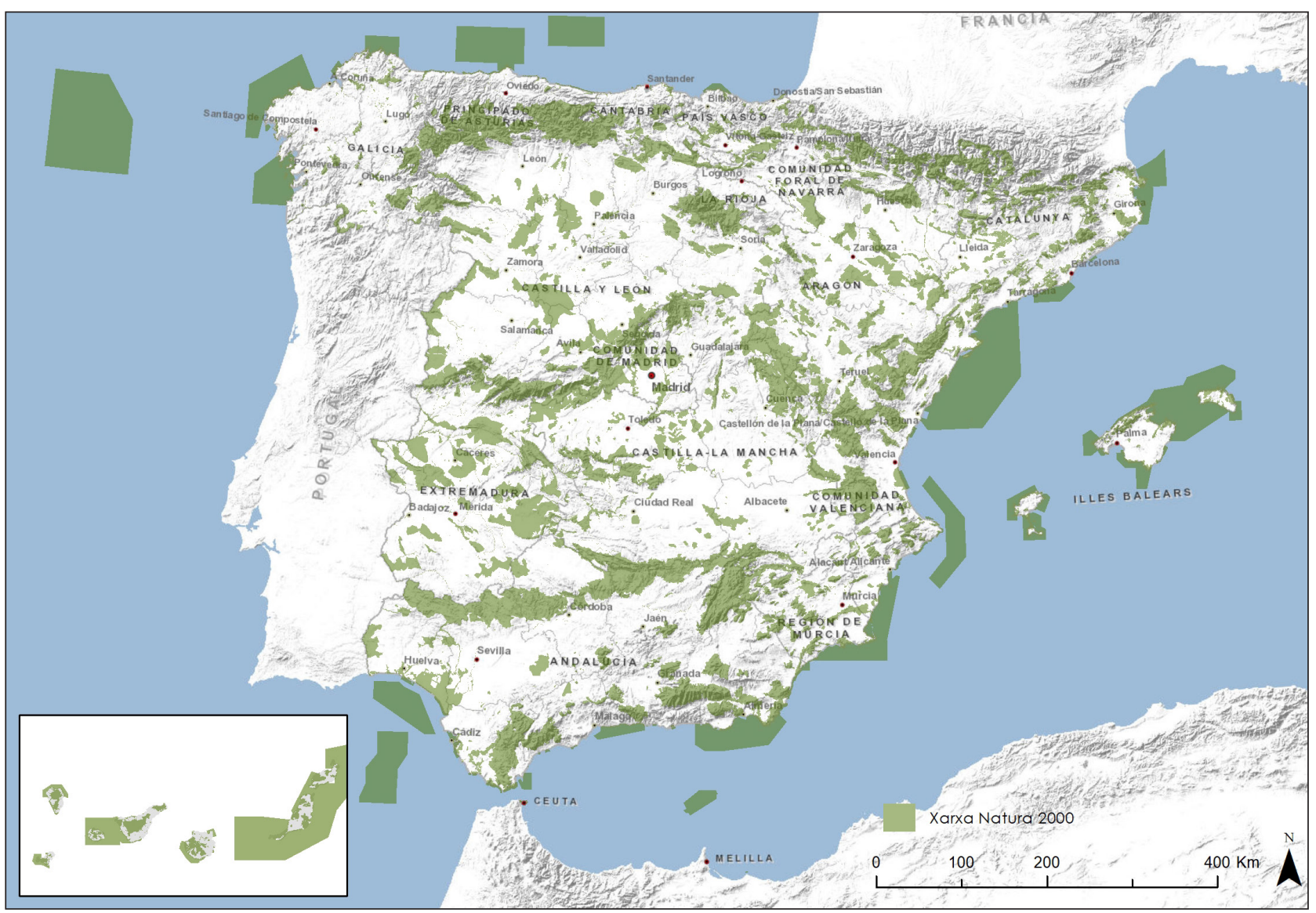

Figura 1: Red Natura 2000. Fuente: Elaboración propia Figure 1: Natura 2000. Source: Own elaboration.

ta en el cálculo de incidencia y caracterización de las Carreras de Trail running y Marchas por montaña en Red Natura 2000.

- Fase 1. Inclusión en la base de datos runedia.com de los siguientes campos: Tipología de evento y Número de edición (elaborados a partir del nombre del evento o a través de la verificación en la web oficial del mismo); Comunidad Autónoma (establecido a partir del campo inicial de provincia); Código de municipio (identificado a partir del campo inicial de población con referencia a la base de datos del Instituto Nacional de Estadística -Código INE).

- Fase 2. Filtrado de la totalidad de eventos disponibles mediante la identificación y eliminación de todos aquellos eventos deportivos no incluidos dentro de las modalidades de Carreras de Trail running y Marchas por montaña. Ejemplo: triatlón, duatlón, etc.

- Fase 3. Georeferenciación del código INE sobre la base cartográfica Red Natura 2000, incluida la consideración de la realización de más de un evento en el mismo municipio.

- Fase 4. Identificación de la distancia óptima Buffer. Esto fue, aplicación de Buffers 3, 5 y 7 km so- bre Red Natura 2000, selección aleatoria de 50 casos de la base de datos, descarga de los correspondientes recorridos o tracks y análisis de la casuística respecto a la incidencia en Red Natura 2000.

- Fase 5. Cálculo de incidencia sobre Red Natura 2000 en base a la consideración del Buffer óptimo, que en este caso fue establecido en $5 \mathrm{~km}$ (margen de error de $+5 \%$, calculado en base al análisis de caso y diferencias con Buffer inferior y superior)

- Fase 6. Identificación de características principales de las carreras y marchas por montaña con y sin incidencia sobre Red Natura 2000.

\subsection{Análisis de datos}

Tal y como se desprende de las fases anteriormente referenciadas, dos fueron los programas utilizados en el desarrollo del presente estudio: SPSS, versión 20.0, en el caso del análisis e identificación de las características principales de los diferentes eventos deportivos analizados, y ARGIS, versión 10.1, en el caso del cálculo de los niveles de incidencia de los eventos sobre Red Natura 2000 . 


\section{Resultados}

\subsection{Tipología de evento, número de edición y distribución territorial}

El análisis descriptivo de la distribución y frecuencia de la tipología y número de edición de los eventos deportivos analizados revelan un claro predominio de la tipología de Carreras de Trail running, con un 76,4\% (incluidas disciplinas tales como trails, carreras en línea, ultras, cros country, km verticales, maratones y medias maratones), seguidos, con valores ciertamente próximos, las tipologías de Marchas por montaña $(13,3 \%)$ y Caminatas $(10,4 \%)$ (Tabla 1).

Respecto al número de edición, tal y como se desprende de la Tabla 2, más del $43 \%$ de las Carreras de Trail running y las Marchas por montaña desarrolladas durante el año 2015 en el territorio español se encuentran incluidas dentro de sus primeras cinco ediciones, reflejando, de esta manera, la presencia de una tasa de crecimiento anual durante estos últimos 5 años en torno al 8,5\%.

Finalmente, del análisis pormenorizado de la distribución de las pruebas deportivas sobre el territorio nacional se evidencia la presencia de una distribución ciertamente heterogénea. Con 819 pruebas, que representan más del 43\% de las Carreras de Trail running y Marchas por montaña desarrolladas en el territorio nacional, Cataluña se constituye como la CCAA mayor

Tabla 1: Tipología de eventos o pruebas deportivas.

Table 1: Sport activity typology.

\begin{tabular}{|l|c|c|}
\hline \multicolumn{1}{|c|}{ Tipología } & Número de eventos & $\mathbf{\%}$ \\
\hline Carreras de Trail running & 1452 & 76,4 \\
\hline Marchas por montaña & 195 & 13,3 \\
\hline Caminatas & 198 & 10,4 \\
\hline Travesías y rutas & 56 & 2,9 \\
\hline
\end{tabular}

Tabla 2: Número de edición.

Table 2: Edition number:

\begin{tabular}{|l|c|c|}
\hline \multicolumn{1}{|c|}{ Edición } & Número de eventos & \% \\
\hline Primera edición & 87 & 4,6 \\
\hline Segunda edición & 178 & 9,4 \\
\hline Tercera edición & 226 & 11,9 \\
\hline Cuarta edición & 187 & 9,8 \\
\hline Quinta edición & 142 & 7,5 \\
\hline Sexta edición & 106 & 5,6 \\
\hline Séptima edición & 89 & 4,7 \\
\hline Octava edición & 68 & 3,6 \\
\hline Novena edición & 52 & 2,7 \\
\hline Más de diez ediciones & 38 & 2,0 \\
\hline Total & $\mathbf{1 9 0 1}$ & $\mathbf{1 0 0}$ \\
\hline
\end{tabular}

número de eventos, seguida, con una importante diferencia, por las CCAA de Valencia y Andalucía, con $180(9,5 \%)$ y 136 pruebas $(7,2 \%)$ respectivamente (Tabla 3).

\subsection{Incidencia de Carreras de Trail running y Marchas por montaña en Red Natura 2000}

Respecto al cálculo de la incidencia de las Carreras de Trail running y Marchas por montaña sobre Red Natura 2000, los datos obtenidos a partir de la georeferenciación del municipio principal del evento y la aplicación de un Buffer de $5 \mathrm{~km}$ alrededor del mismo, constatan un nivel de incidencia ciertamente elevado. Más del $85 \%$ de las Carreras de Trail running y Marchas por montaña desarrolladas en el territorio español durante el año 2015 tienen incidencia sobre algunos de los ENP pertenecientes a Red Natura 2000; concretamente, 1635 eventos incluyeron dentro de su recorrido/s superficie de esta red de Conservación (Figura 2).

Ahora bien, dentro de esta línea, pero desde una perspectiva más intra Comunidad, es decir, análisis de la incidencia de los eventos deportivos dentro de las diferentes CCAA, se evidencia la presencia de algunos datos de interés, sobre todo si tenemos en cuenta que el valor medio de incidencia intra Comunidad se sitúa en torno al $72 \%$. Por ejemplo, mientras que Ceuta, Extremadura e Islas Ba-

Tabla 3: Distribución por Comunidades autónomas.

Table 3: Territorial distribution.

\begin{tabular}{|l|c|c|}
\hline Comunidad Autónoma & Total eventos & \% \\
\hline Catalunya & 819 & 43,1 \\
\hline Com. Valenciana & 180 & 9,5 \\
\hline Andalucía & 136 & 7,2 \\
\hline Aragón & 99 & 5,2 \\
\hline Galicia & 94 & 4,9 \\
\hline Canaríes & 93 & 4,9 \\
\hline Castilla y León & 91 & 4,8 \\
\hline País Vasco & 64 & 3,4 \\
\hline Comunidad de Madrid & 58 & 3,1 \\
\hline Cantabria & 53 & 2,8 \\
\hline Asturias & 44 & 2,3 \\
\hline Islas Baleares & 40 & 2,1 \\
\hline Extremadura & 36 & 1,9 \\
\hline Castilla la Mancha & 31 & 1,6 \\
\hline Comunidad de Navarra & 24 & 1,3 \\
\hline Murcia & 23 & 1,2 \\
\hline La Rioja & 15 & 0,8 \\
\hline Ceuta & $\mathbf{1 9 0 1}$ & 0,1 \\
\hline Total & & $\mathbf{1 0 0}$ \\
\hline
\end{tabular}




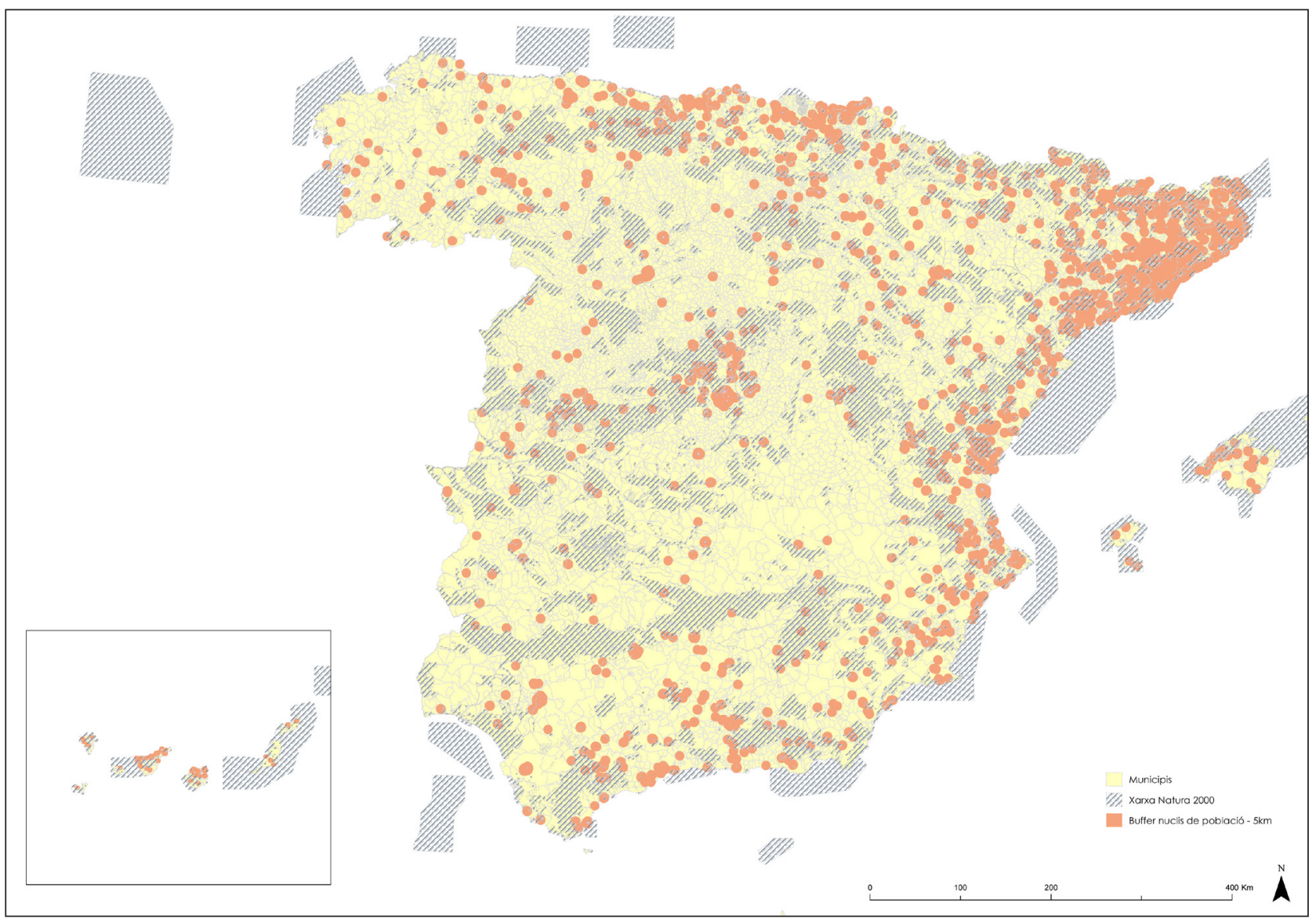

Figura 2: Incidencia carreras y marchas por montaña Red Natura 2000. Fuente: Elaboración propia.

Figure 2: Trail running impact in Natura 2000. Source: Own elaboration.

leares presentan un porcentaje de incidencia superior al 95\%; País Vasco, con 64 eventos deportivos, apenas supera el $51 \%$ de incidencia (Tabla 4 ).

Ahora bien, en lo que respecta a la evolución de la incidencia de este tipo de eventos sobre Red Natura 2000, en la Figura 3 se puede constatar que su evolución no presenta grandes diferencias en relación al conjunto de eventos de la Península.

\subsection{Características especificas de las Carreras de Trail running y Marchas por montaña desarrolladas en Red Natura 2000}

Respecto a datos relacionados con la implementación de este tipo de eventos, es decir, características que pueden tener una mayor implicación sobre el tipo y el nivel de los impactos ambientales asociados o problemáticas de gestión vinculadas (otorgamiento de autorizaciones, etc.), los resultados obtenidos revelan la presencia de ciertos rasgos característicos de gran importancia si a la gestión sostenible de este tipo de eventos hacemos referencia (minimización de impactos ambientales negativos, ampliación de impactos socioeconómicos positivos).
Aunque la temporada de primavera es uno de los meses más críticos en ciertos aspectos de la conservación de este tipo de espacios, nidificación y reproducción de las aves, por ejemplo, más del $35 \%$ de los eventos deportivos que transcurrieron por algunos de los ENP incluidos en Red Natura 2000 fueron llevados a cabo en esta temporada. Por el contrario, y en este caso aparentemente a favor de la minimización de impactos (minimización del área ocupada o número de participantes), más del $70 \%$ de los eventos deportivos que tienen lugar en estos espacios implementaron un solo recorrido o track (Tablas 5 y 6).

\section{Discusión y conclusiones}

Los resultados expuestos a lo largo de este trabajo, no solo caracterizan de forma genérica el conjunto de Carreras de Trail running y Marchas por montaña desarrolladas en España durante el año 2015, sino que plantea una primera propuesta de cálculo del nivel de incidencia de estas sobre los diferentes ENP incluidos en Red Natura 2000, lo que lo convierte en un estudio pionero y novedoso que aporta una serie de datos que creemos relevantes en el campo de la organización de eventos deportivos y de la conservación del medio natural. 
Tabla 4: Incidencia Red Natura 2000 por Comunidades autónomas.

Table 4: Natura 2000 territorial impact.

\begin{tabular}{|l|c|c|c|}
\hline \multicolumn{1}{|c|}{ Comunidad Autónoma } & Total eventos & Eventos con incidencia & Incidencia por CCAA \\
\hline Ceuta & 1 & 1 & $100,0 \%$ \\
\hline Extremadura & 36 & 35 & $97,2 \%$ \\
\hline Islas Baleares & 40 & 38 & $95,0 \%$ \\
\hline Canarias & 93 & 88 & $94.6 \%$ \\
\hline Cataluña & 819 & 755 & $92.2 \%$ \\
\hline Murcia & 23 & 21 & $91,3 \%$ \\
\hline Comunidad de Madrid & 58 & 51 & $87,9 \%$ \\
\hline Aragón & 99 & 86 & $86,8 \%$ \\
\hline Com. Valenciana & 180 & 153 & $85,0 \%$ \\
\hline Castilla La Mancha & 31 & 26 & $83,8 \%$ \\
\hline Asturias & 44 & 36 & $81,8 \%$ \\
\hline Castilla y León & 91 & 73 & $80,2 \%$ \\
\hline La Rioja & 15 & 12 & $80,0 \%$ \\
\hline Cantabria & 53 & 41 & $77,3 \%$ \\
\hline Andalucía & 136 & 102 & $75,0 \%$ \\
\hline Galicia & 94 & 68 & $72,3 \%$ \\
\hline Comunidad de Navarra & 24 & 16 & $66,6 \%$ \\
\hline País Vasco & $\mathbf{1 9 0 1}$ & 33 & $51,5 \%$ \\
\hline Total & & $\mathbf{1 6 3 5}$ & -- \\
\hline
\end{tabular}

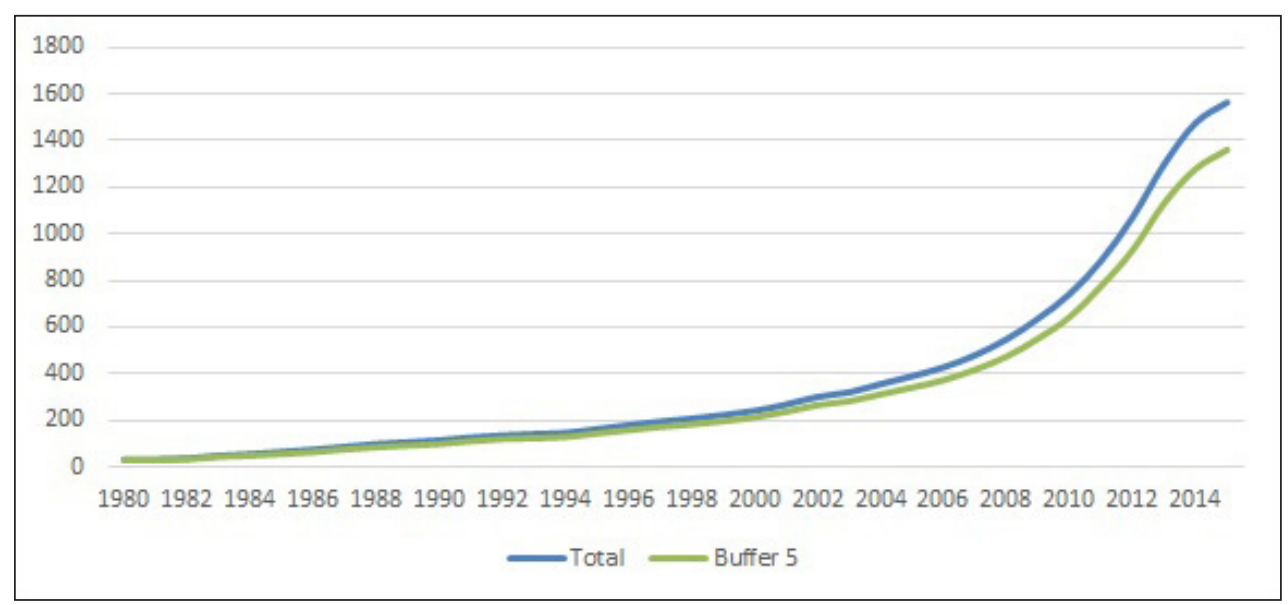

Figura 3: Número y evolución Carreras de Trail running y Marchas por montaña España y Red Natura 2000.

Figure 3: Evolution of Trail running races in Natura 2000.

Mediante el análisis y sistematización de un total de 1901 eventos deportivos organizados durante el año 2015, los resultados obtenidos permiten avanzar en el conocimiento de la tipología, evolución y distribución territorial de estos en España, en el establecimiento del grado de incidencia sobre Red Natura 2000 y en la identificación de algunas de las características principales de las Carreras de Trail running y Marchas por montaña que transcurren en algunos de los ENP de Red Natura 2000.

En primer lugar, y en relación a la tipología, evolución y distribución territorial de estos eventos, cabe destacar, que los resultados obtenidos revelan que la mayoría de eventos se corresponden a la tipología de carreras a pie, se sitúan dentro de sus primeras cinco ediciones y se concentran, de forma realmente llamativa, en la CCAA de 
Tabla 5: Distribución por meses del año.

Table 5: Trail running races month distribuition

\begin{tabular}{|l|c|c|}
\hline \multicolumn{1}{|c|}{ Meses } & Número de eventos & \% \\
\hline Enero & 43 & 2,6 \\
\hline Febrero & 74 & 4,5 \\
\hline Marzo & 159 & 9,7 \\
\hline Abril & 176 & 10,8 \\
\hline Mayo & 232 & 14,2 \\
\hline Junio & 175 & 10,7 \\
\hline Julio & 118 & 7,2 \\
\hline Agosto & 98 & 6,0 \\
\hline Septiembre & 161 & 9,8 \\
\hline Octubre & 201 & 12,3 \\
\hline Noviembre & 152 & 9,3 \\
\hline Diciembre & 46 & 2,8 \\
\hline Total & $\mathbf{1 6 3 5}$ & $\mathbf{1 0 0}$ \\
\hline
\end{tabular}

Tabla 6. Número de recorridos contemplados en los diferentes eventos.

Table 6. Number of tracks in each trail running race.

\begin{tabular}{|l|c|c|}
\hline Número de recorridos & Número de eventos & $\mathbf{\%}$ \\
\hline 1 recorrido & 1281 & 78,3 \\
\hline 2 recorridos & 273 & 16,7 \\
\hline 3 recorridos & 62 & 3,8 \\
\hline Más de tres & 19 & 1,2 \\
\hline Total & $\mathbf{1 6 3 4}$ & $\mathbf{1 0 0}$ \\
\hline
\end{tabular}

Cataluña. Concentración, que en este sentido, podría ser justificada por circunstancias tales como: el nivel de asociacionismo histórico presente en esta Comunidad, en muchos casos, pieza clave en el impulso y organización de este tipo de eventos (Burriel \& Puig, 1999), los hábitos deportivos de la población, históricamente también superiores al resto de CCAA y, especialmente, en el ámbito de la montaña (García Ferrando \& Llopis, 2010; Puig et al., 2014), y por qué no, a la cultura propia de esta Comunidad, caracterizada por un dinamismo social elevado y una amplia capacidad de identificar oportunidades de negocio (Guallarte et. al., 2016). Aspectos, todos estos, que cabría analizar y profundizar con más detalle si en el estudio de estas circunstancias se quiere profundizar.

En segundo lugar, y respecto al nivel de incidencia sobre Red Natura 2000, destaca la identificación de una incidencia superior al $85 \%$, con unos niveles de incidencia intra comunidad ciertamente dispares. Destaca, en este sentido, la reducida incidencia intra Comunidad presente, por ejemplo, en el País Vasco. Mientras que la media de incidencia intra comunidad para el conjunto de CCAA se sitúa en torno al $72 \%$, el País Vasco apenas supera el $51 \%$. Analizar el porqué, y, si cabe, los mecanismos exis- tentes en la administración de los ENP de esta CCAA en torno a la concesión de autorizaciones y/o gestión activa de sus territorios, podrían facilitar el camino a aquellas CCAA con una presión ciertamente preocupante, en volumen e incidencia, como lo son las CCAA de Extremadura, Islas Baleares, Canaria o Cataluña, con niveles de incidencia intracomunitaria superiores al $92 \%$.

En tercer y último lugar, y en lo que se refiere a la identificación de algunas de las características principales de las Carreras de Trail running y Marchas por montaña desarrolladas en Red Natura 2000, destaca un crecimiento paralelo al conjunto de eventos, una mayor concentración durante los meses de primavera y octubre, probablemente atendiendo a las características del clima Mediterráneo y al calendario de competición de algunas de las disciplinas incluidas dentro de este tipo de eventos. Al respecto, resulta evidente la problemática que puede derivarse de este dato teniendo en cuenta que algunos de estos meses pueden coincidir con episodios de máxima floración y crecimiento, a nivel de flora, y/o cría o nidificación, a nivel de fauna, (Oñorbe, 2014b).

En términos de aplicación, y remitiéndonos a los ámbitos de actuación considerados en la Guía de Buenas Prácticas para el desarrollo de carreras por montañas en Espacios Naturales Protegidos (Europarc-España, 2016): entidades organizadoras; administraciones responsables y participantes, hay que destacar las siguientes implicaciones prácticas:

Los resultados obtenidos en relación a la distribución anual de carreras de Trail Running y Marchas por montaña muestran un claro predominio de eventos durante los meses más críticos. El establecimiento de un calendario global por parte de las administraciones de los diferentes espacios, y la redistribución de los diferentes eventos por parte de las entidades organizadoras deberían ser hoy por hoy una de las acciones prioritarias a implementar. No sirve, en este sentido, un calendario global impuesto por parte de las administraciones responsables, sino un consenso caso a caso de la necesidad de redireccionar el calendario global de competiciones por parte de las diferentes entidades organizadoras, con el correspondiente entendimiento de estas necesidades por parte de los participantes (informar sobre la necesidad de cambios de fechas, etc.). Explicar el qué y por qué resulta imprescindible si lo que se quiere es avanzar hacia la compatibilización de usos (Farías \& Sallent, 2009). Casos de estudio y ejemplos prácticos podrían ayudar en la implementación de estos cambios.

Así mismo, de forma contraria a algunas de las preocupaciones manifiestas por algunas entidades gestoras respecto al número de recorridos incluidos en los diferentes eventos, los datos obtenidos en este estudio no sustentan la necesidad de considerar este aspecto como un punto crítico a abordar. Más del 78\% de los eventos deportivos que tienen incidencia sobre espacios de Red Natura 2000 solo contemplan un recorrido. Sin embargo, no por minoritario deja de ser un aspecto a continuar observando, sobre todo si nos remitimos a una tendencia clara de convertir estas pruebas deportivas como eventos cada vez más populares, con una oferta de distancias más acorde a los diferentes pú- 
blicos. Una vez más, el estudio caso a caso de los eventos históricamente desarrollados, y la implementación de unas reglas de juego claras de futuro, parecen ser, en este sentido, una de las escasas herramientas de negociación disponibles por parte de las administraciones responsables y entidades organizadoras. Al respecto, hay que destacar una de las iniciativas recientemente implementadas en el Parque Natural de la Serra de Collserola (establecimiento de una red principal de pistas y senderos a utilizar en la organización de este tipo de eventos), que bien puede constituirse como un caso de estudio a observar detenidamente.

\section{Limitaciones y perspectivas de futuro}

En este estudio, la muestra analizada se basó en la consideración de una sola fuente de información, 1901 eventos deportivos, por lo que reconocemos, claramente, la limitación que este hecho comporta. Asumimos, sin lugar a dudas, la imposibilidad de considerar dicha cifra como un dato totalmente fiable, dando por hecho que existe un número desconocido de carreras no censadas. Así, se debería mejorar el número de fuentes y la sistematización de la recogida de algunos datos, como lo pueden ser, el detalle de los diferentes recorridos o tracks. La falta de detalle de los tracks de los diferentes recorridos es un aspecto de gran importancia que queda pendiente abordar en un estudio más amplio y sistemático de casos. Esto es, intentar diferenciar la coincidencia o no de los recorridos incluidos en los recorridos, establecer un valor lo más exacto posible de la distancia o porcentaje de recorrido/s que transcurre a lo largo de los diferentes ENP.

\section{Referencias}

Bataller, V., Macén, C., Piedrafita, E. \& Arbonés, I., 2014. Comparación de los perfiles de corredores de carreras populares y corredores de carreras por montaña. En: Crisis, cambio social y deporte. XIII Congreso Internacional AEISAD Investigación Social y Deporte 12. http://www.zaragozadeporte.com/docs/Boletin/documento5133.pdf.

Burgin, S. \& Hardiman, N., 2012. Extreme sports in natural areas: Looming disasteror a catalyst for a paradigm shift in land use planning? Journal of Environmental Planning and Management, 55(7): 921-940. https://doi.org/10.1080/0964 0568.2011.634228

Burriel, J. \& Puig, N., 1999. Responsabilidades y relaciones entre el sector público y el privado en el sistema deportivo. En Subirats, J. (Editor) ¿Existe sociedad civil en España? Responsabilidades colectivas y valores públicos. Fundación Encuentro. Madrid.

Cater, R., Buckley, R., Hales, D., Newsome, D., Pickering, C \& Smith, A., 2008. High impact activities in parks: best management practice and future research. Gold Coast. Queensland: Cooperative Research Centre for Sustainable Tourism, Griffith University.

Cole, D. 1990. Ecological impacts of wilderness recreation and their management. In: Hendee, C., et al. (eds.). Wilderness Management. Stewardship and protection of resources and values. 3rd ed. Colorado: Golden, pp. 425-462.
EUROPARC-ESPAÑA, 2016. Guia de buenas prácticas para el desarrollo de carrera por montaña en espacios protegidos. Europarc-España, Madrid. http://www.fedme.es/index.php? mmod $=$ salaprensa\&file $=$ list $\& \mathrm{cID}=14$

Ewert, A., Attarian, A., Hollenhorst, S., Russell, K. \& Voight, A., 2006. Evolving adventure pursuits on public lands: emerging challenges form management and public policy. Journal of park and Recreation Administration, 24 (2): 125-140. https:// js.sagamorepub.com/jpra/issue/view/180

Farías, E. \& Sallent, O., 2009. El impacto ambiental de las actividades físico-deportivas en el medio natural. El caso de la práctica del Moutain Bike o bicicleta todo terreno. Revista Retos. Nuevas tendencias en Educación Física, Deporte y Recreación, 16: 31-35.

Farías, E., 2015. Minimización de los impactos medioambientales negativos en la organización de eventos deportivos en el medio natural. El caso de las Marchas de Bicicleta todo terreno (BTT) o Mountain Bike (MTB). Revista Apunts, 122 (4T): 68-80. http://dx.doi.org/10.5672/apunts.2014-0983. cat.(2015/4).122.08

Farías, E., Seguí, J. \& Indres, E., 2015. Profiling participants of sporting events in the natural environment: trail running races. En: Sustainable development of sport tourism. Plevnik, M., Reta, I., Pisot, R. \& Obid, A., Edts. Annals Universit Pres. Cooper. 185-195 pp. http://www.zrs-kp.si/monografije/single/sustainbale-development-of-sports-tourism-1902

Farías, E., Seguí, J., Mena, X. \& Sabaté, A., 2016. Eventos deportivos en espacios naturales protegidos. El caso de la red de Espacios de Protección Espacial de Catalunya. Boletin Europarc España. Revista técnica de los espacios naturales protegidos, 43: 28-31

FEDME (Federación Española de Deportes de Montaña y Escalada), 2011. Incidencia socioeconómica y ambiental de las carreras por montaña en el medio natural y rural en España. Madrid. http://www.fedme.es/salaprensa/upfiles/9_F_es.pdf

Fernández-Arroyo, R., 2015. Cuando correr en montaña deja de ser un deporte amable. Quercus, 347: 78-81.

Fundació Mon Rural i Centre Tecnològic Forestal de Catalunya, 2017. Codi de bones pràctiques en l'organització i celebració de curses i marxes per muntanya. http://www.fmr.cat/sites/default/files/adjunts-fmr/cobo_bones_practiques_ baixa_0.pdf

García Ferrando, M. \& Llopis, R., 2010. Ideal democrático y bienestar personal: Encuesta sobre los hábitos deportivos en España. Consejo Superior de Deportes. 268 pp.

Granero, A. \& Baena, A., 2010. The search for nature as a way of compensation of the new urban lifestyle. Journal of Sport and Health Research, 2(1): 17-25.

Guallarte, C., Capelleres, J., Fiuls, M., Genescá, E. \& Obis, T., 2016. Global Entreprenership Monitor. Informe executiu Catalunya 2015. http://www.gem-spain.com//wp-content/ uploads/2015/03/GEM-Cataluna-2015.pdf

Leung, Y. \& Marion, J., 2000. Recreation Impacts and Management in Wilderness: A State-of-Knowledge Review. USDA Forest Service Proceedings RMRS-P-15, 5:23-48. https://www. fs.fed.us/rm/pubs/rmrs p015 5/rmrs p015 5 023 048.pdf

Martos, P., 2002. El Medio Natural como Punto de Encuentro de Turismo y Deporte. Crecimiento y Diversificación. In: S. Rebollo; M. D. Simoes (Eds.): Nuevas Tendencias de Práctica Físico-Deportiva en el Medio Natural. Granada: Universidad de Granada, 3-14 pp.

Newsome, D., Lacroix, C. \& Pickering, C., 2011. Adventure racing events in Australia. Context, assessment and implications for protected area management. Australian Geographer, 42 (4): 403-418. https://doi.org/10.1080/00049182.2012.619955

Newsome, D. \& Lacroix, C., 2012. A research agenda for adventure racing events that take place in natural settings and protected areas. In: Outdoor recreation in change - current 
knowledge and future challenges. 6th International Conference on Monitoring and Management of Visitors in Recreational and Protected Areas. Stockholm, Sweden, August: 21-24. http://miun.diva-portal.org/smash/get/diva2:580065/ FULLTEXT01.pdf

Newsome, D., 2014. Appropriate policy development and research needs in response to adventure racing in protected areas. Biological Conservation 171: 259-269. https://doi. org/10.1016/j.biocon.2014.01.008

Oñorbe, M., 2014a. Los retos en la gestión de las actividades deportivas en Espacios Naturales Protegidos. Congreso $\mathrm{Na}$ cional del Medio Ambiente. COMANA 2014. http://www. conama.org/conama/download/files/conama2014/CT\%20 2014/1896711249.pdf

Oñorbe, M., 2014b. Evaluación impacto ambiental carreras por la montaña. Carrera de montaña Demandafolk (Sierra de la Demanda, Burgos). Territorios vivos. Recuperado de: http://manuelmedioambiente.wordpress.com/2014/02/24/ evaluacion-ambiental/.
Oñorbe, M., 2015. Guía orientativa para la evaluación ambiental de carreras por montaña que pueden afectar a espacios de Red Natura 2000 en Canarias. http://issuu.com/festucaelegans/docs/guia_ambiental_cxm_canarias_borrado

Pickering, C., 2010. Ten factors that affect the severity of environmental impacts of visitors in protected areas. Ambio, 39:70-77. https://doi.org/10.1007/s13280-009-0007-6

Puchan, H., 2005. Living extreme: adventure sports, media and commercialization. Journal of Communication Management, 9 (2):171-178. https://doi.org/10.1108/13632540510621588

Puig, N., Munoz, L., Camps, A., Gomez, J., Serra, P \& Pardo, A., 2014. L'esport a Catalunya. Un informe de tendències (20062013). Observatori Català de l' Esport http://www.observatoridelesport.cat/docus/info esport/OCE 3 info_esport_es.pdf

Seguí, J., Ingles, E., Labrador, V. \& Farías, E., 2016. Carreras (de o por) montaña o trail running. El reconocimiento de la modalidad deportiva: una visión jurídica. Revista Retos. Nuevas Tendencias en Educación Física, Deporte y Recreación (25): 162-167. 